Nota técnica

\title{
Registros documentados del Chirigüe Azafranado (Sicalis flaveola Linnaeus, 1766; Aves: Thraupidae) en Loreto, Perú
}

\author{
[Documented records of Saffron Finch (Sicalis flaveola Linnaeus, 1776; \\ Aves: Thraupidae) in Loreto, Peru]
}

\author{
Luis Armando Garcia Solsol ${ }^{* 1}$, Gino Anthony Tuesta Cometivos ${ }^{1}$, Freddy Robert Ferreyra Vela², \\ Luis Alfonso Bernuy Pinedo ${ }^{1}$, Juan Díaz Alván ${ }^{3}$ \\ 1. Universidad Nacional de la Amazonía Peruana (UNAP). Facultad de Ciencias Biológicas. Pevas 5tacuadra, \\ Iquitos, Maynas, Loreto, Perú. Correos electrónicos: luisgarciasolsol@gmail.com \\ (L. A. Garcia * Autor para correspondencia), ginoanthony29@gmail.com (G. A. Tuesta), \\ luiskazuto123@gmail.com (L. A. Bernuy). \\ 2. Instituto del Bien Común (IBC). Calle Nauta 334. Iquitos, Maynas, Loreto, Perú. \\ Correo electrónico: fferreyra@ibcperu.org (F. R. Ferreyra).
}

3. Universidad Científica del Perú (UCP). Av. A. Quiñones km 2,5 (Iquitos), San Juan Bautista, Maynas, Loreto, Perú. Correo electrónico: jdiazalvan@gmail.com (J. Díaz).

\section{Resumen}

El Chirigüe Azafranado (Sicalis flaveola) es una especie bastante común en la parte Noroeste, costa del Perú y frecuente en algunas ciudades. Documentamos la presencia de $S$. flaveola en el departamento Loreto, con lo cual se amplía su distribución en Perú, adicionalmente se proporciona algunos datos sobre su comportamiento, número de individuos y residencia.

Palabras clave: Actividad antrópica, Ampliación de rango, Registro en Loreto, Residente.

\begin{abstract}
The Saffron Finch (Sicalis flaveola) is a fairly common species in the northwestern and coastal parts of Peru, frequently in cities. We document the presence of $S$. flaveola in the department of Loreto, consequently expanding its distribution in Peru. Additionally, we provide behavioral data as well as number of individuals and residence.
\end{abstract}

Keywords: Anthropic activity, Range extension, Record in Loreto, Resident. 


\section{INTRODUCCIÓN}

El Chirigüe Azafranado (Sicalis flaveola), es un ave inconfundible por su coloración amarilla, los machos se caracterizan por presentar una corona amarillo rojizo. Se considera común, y está presente en gran parte de América del Sur, especialmente en Ecuador, Colombia, Perú, Venezuela, Guyanas, Bolivia, Brasil, Paraguay, Argentina, Uruguay. Se conoce que la especie ha sido introducida en algunas ciudades de $\mathrm{Pa}$ namá, Jamaica, Puerto Rico y Chile, así como también en algunas ciudades de Perú (Ruiz, 2002, 2007; Buitrón y Freile, 2006; Paetzold y Querol, 2008; Guilherme, 2011; Rising y Jaramillo, 2020). S. flaveola se encuentra catalogada como en Preocupación Menor (LC) (IUCN, 2021) y su población se encuentra estable (Birdlife International, 2021). En general su distribución no sobrepasa los 2200 m de elevación y se le encuentra en una gran variedad de hábitats que van desde áreas abiertas con árboles o arbustos dispersos, bordes de río, bosques secundarios, áreas agrícolas, áreas urbanas, entre otros, alimentándose de semillas y también de pequeños artrópodos (Espinoza et al., 2017; Rising y Jaramillo, 2020). En Colombia la especie se encuentra ampliamente distribuida en la región Caribe, oriente de los Andes desde Arauca hasta Meta y oriente del Vichada, en los llanos colombianos, luego reportada en el valle del Cauca como aves escapadas de jaulas y ahora con registros en el interfluvio del Caquetá-Putumayo y en la ciudad de Leticia, probablemente como consecuencia de aparición de áreas deforestadas (Hilty y Brown, 1986; Espinosa et al., 2017; Acevedo-Charry et al., 2021).

En Perú, S. flaveola es bastante común en el Noroeste, en la costa y en las partes más bajas y semiáridas del valle del Marañón hasta los $2200 \mathrm{msnm}$. Es una especie frecuente en ciudades y jardines, observada en parejas o pequeñas bandadas y comúnmente mantenida en cautiverio, el cual al escaparse de jaulas pueden ser encontradas en pueblos y ciudades lejos de su rango de distribución original (Schulenberg et al., 2010; Silva et al., 2012), razón por la cual fueron introducidos en la ciudad de Lima y Puerto Maldonado en el departamento de Ma- dre de Dios, Perú (Guilherme, 2011; Guevara, 2012; Nolazco, 2012; Begazo, 2021).

En esta nota científica se documenta la presencia de $S$. flaveola por primera vez en el departamento de Loreto, Perú, dando a conocer algunos datos sobre su permanencia, comportamiento y número de individuos observados en la selva Nororiental.

\section{REGISTROS}

El 28 de febrero del 2020, L. A. Bernuy, observó y fotografió a 1 individuo de $S$. flaveola a las 06 horas y 45 minutos (Tabla 1, Figura 2) forrajeando en algunas áreas con hierbas gramíneas y ciperáceas que crecen en la zona baja del boulevard de la ciudad de Iquitos, departamento de Loreto, Perú (Figura 1); el ave observada era inconfundible por la coloración amarilla y la corona amarilla rojiza, características de la especie.

El 28 de octubre del 2020, L. A. Garcia, G. A Tuesta y J. Díaz, observaron y fotografiaron a un individuo de $S$. flaveola a las 9 horas con 17 minutos (Figura 3), transportando hierbas secas en el pico, probablemente con la intención de construir nido, el hecho tuvo lugar en la parte baja del boulevard de Iquitos, en temporada de aguas bajas de los ríos.

El 29 de octubre del 2020, J. Díaz, L. A. Garcia y G. A Tuesta, observaron y fotografiaron un individuo de $S$. flaveola a las 08 horas y 10 minutos, en los alrededores del boulevard de Iquitos, se encontraba perchado en un cable de alumbrado público y posteriormente se desplazó hacia la zona baja del boulevard, probablemente en busca de alimento.

El 04 de noviembre del 2020, L. A. Garcia, G. A Tuesta y J. Díaz, observaron a un individuo de S. flaveola a las 06 horas y 53 minutos (Figura $4 \mathrm{~A}$ ), en los cables de luz que se encuentran en los alrededores del boulevard, con un comportamiento de defensa frente a las agresiones de un individuo de Tyrannus albogularis. 




Figura 1. Mapa de registros de Chirigüe Azafranado (Sicalis flaveola) en Loreto, Perú.

Tabla 1. Registros del Chirigüe Azafranado (Sicalis flaveola) en Loreto, Perú.

\begin{tabular}{|c|c|c|c|c|c|c|c|}
\hline $\mathbf{N}^{\circ}$ & Observadores & $\begin{array}{c}N^{\circ} \text { de } \\
\text { indivi- } \\
\text { duos }\end{array}$ & Fechas & $\begin{array}{c}\text { Hora de } \\
\text { obser- } \\
\text { vación }\end{array}$ & Lugar de observación & Coordenadas & $\begin{array}{l}\text { Eleva- } \\
\text { ción }(\mathrm{m})\end{array}$ \\
\hline 1 & L. A. Bernuy & 1 & $28 / 02 / 2020$ & 06:45 & Boulevard de Iquitos & $\begin{array}{l}03^{\circ} 45^{\prime} \quad 1.6^{\prime \prime} \mathrm{S} ; \\
73^{\circ} 14^{\prime} 32.4^{\prime \prime} \mathrm{W}\end{array}$ & 81 \\
\hline 2 & $\begin{array}{l}\text { L. A. Garcia; G. A. Tues- } \\
\text { ta y J. Díaz }\end{array}$ & 1 & $28 / 10 / 2020$ & 09:17 & Boulevard de Iquitos & $\begin{array}{l}03^{\circ} 45^{\prime} \quad 1.6^{\prime \prime} \mathrm{S} ; \\
73^{\circ} 14^{\prime} 32.4^{\prime \prime} \mathrm{W}\end{array}$ & 81 \\
\hline 3 & $\begin{array}{l}\text { J. Díaz; L. A. Garcia y G. } \\
\text { A. Tuesta }\end{array}$ & 1 & $29 / 10 / 2020$ & 08:10 & Boulevard de Iquitos & $\begin{array}{l}03^{\circ} 45^{\prime} \quad 1.6^{\prime \prime} \mathrm{S} ; \\
73^{\circ} 14^{\prime} 32.4^{\prime \prime} \mathrm{W}\end{array}$ & 81 \\
\hline 4 & $\begin{array}{l}\text { L. A. Garcia; G. A. Tues- } \\
\text { ta y J. Díaz }\end{array}$ & 1 & $4 / 11 / 2020$ & 06:53 & Boulevard de Iquitos & $\begin{array}{l}03^{\circ} 45^{\prime} \quad 1.6^{\prime \prime} \mathrm{S} ; \\
73^{\circ} 14^{\prime} 32.4^{\prime \prime} \mathrm{W}\end{array}$ & 81 \\
\hline 5 & $\begin{array}{l}\text { G. A. Tuesta y L. A. Gar- } \\
\text { cia }\end{array}$ & 1 & $11 / 11 / 2020$ & $08: 24$ & Boulevard de Iquitos & $\begin{array}{l}03^{\circ} 45^{\prime} \quad 1.6^{\prime \prime} \mathrm{S} ; \\
73^{\circ} 14^{\prime} 32.4^{\prime \prime} \mathrm{W}\end{array}$ & 81 \\
\hline 6 & $\begin{array}{l}\text { L. A. Garcia y G. A. Tues- } \\
\text { ta }\end{array}$ & 1 & $10 / 01 / 2021$ & 07:00 & Boulevard de Iquitos & $\begin{array}{l}03^{\circ} 45^{\prime} \quad 1.6^{\prime \prime} \mathrm{S} ; \\
73^{\circ} 14^{\prime} 32.4^{\prime \prime} \mathrm{W}\end{array}$ & 81 \\
\hline 7 & F. Ferreyra & 7 & $22 / 04 / 2021$ & 07:00 & $\begin{array}{l}\text { Comunidad Nativa Tres } \\
\text { Esquinas-Bajo Putumayo }\end{array}$ & $\begin{array}{llll}2^{\circ} & 29^{\prime} & 19.6^{\prime \prime} & \mathrm{S} ; \\
70^{\circ} & 35^{\prime} & 50.47^{\prime \prime} & \mathrm{W}\end{array}$ & 78 \\
\hline 8 & F. Ferreyra & 9 & $23 / 04 / 2021$ & 08:00 & $\begin{array}{l}\text { Comunidad Nativa Tres } \\
\text { Esquinas-Bajo Putumayo }\end{array}$ & $\begin{array}{llll}2^{\circ} & 29^{\prime} & 19.6^{\prime \prime} & \mathrm{S} ; \\
70^{\circ} & 35^{\prime} & 50.47 " & \mathrm{~W}\end{array}$ & 78 \\
\hline
\end{tabular}




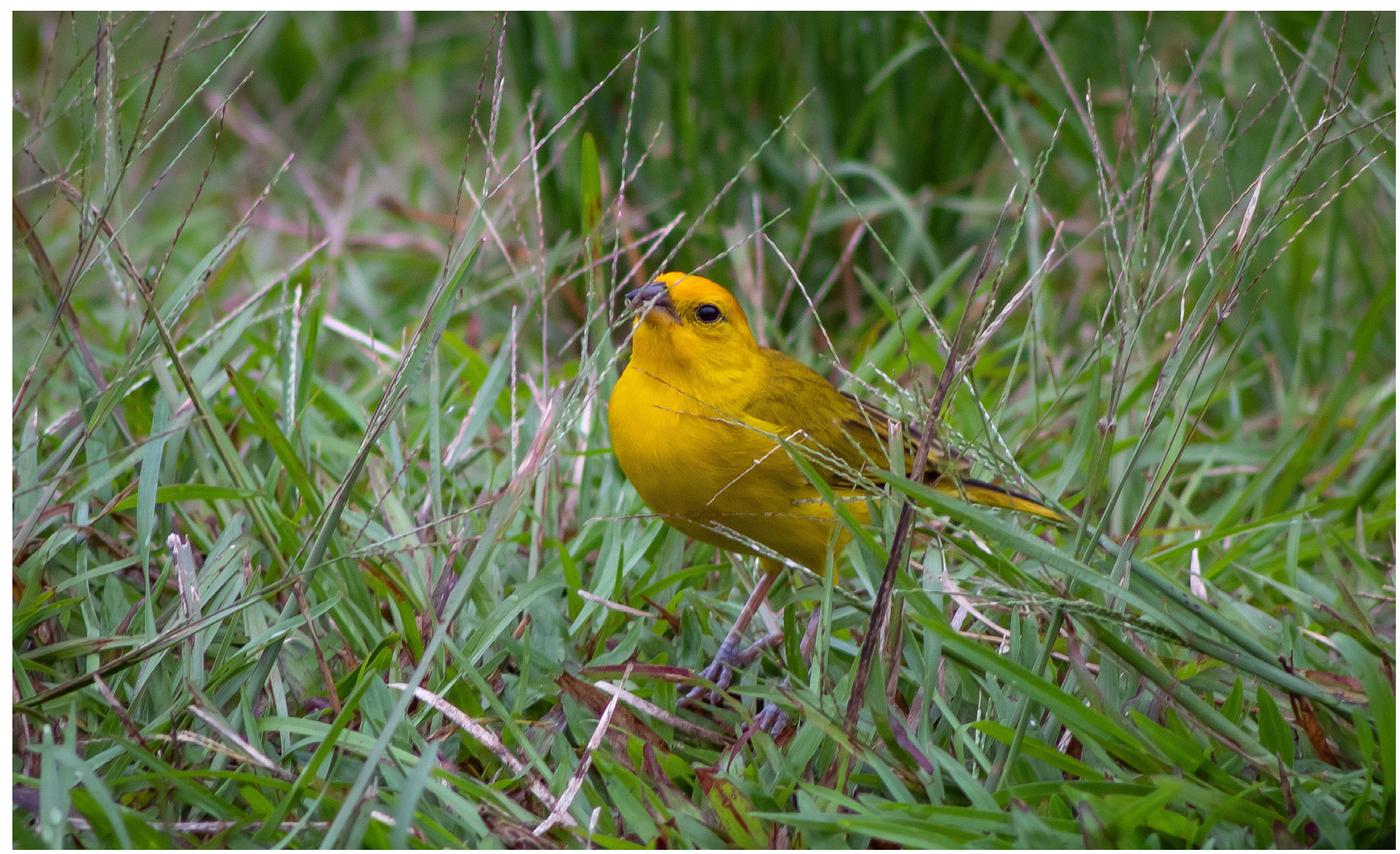

Figura 2. Individuo de Sicalis flaveola en los alrededores del boulevard de Iquitos, Loreto, Perú. Febrero del 2020. Foto: Luis A. Bernuy.



Figura 3. Individuo de Sicalis flaveola, perchado después de haber recolectado restos de hierbas secas en la parte baja del boulevard de Iquitos, Loreto, Perú. Octubre del 2020. Foto: Gino A. Tuesta. 

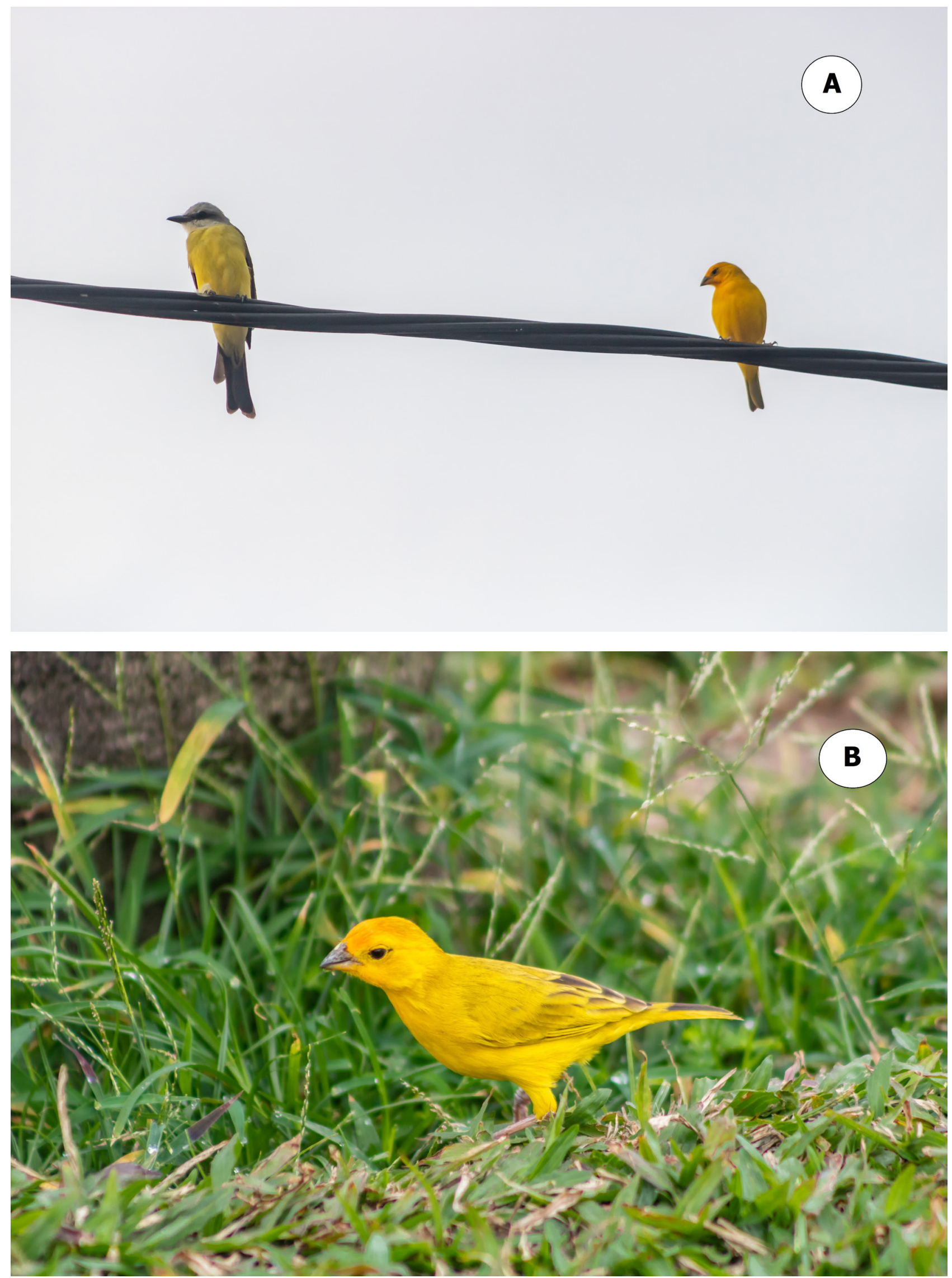

Figura 4. Fotografías de Sicalis flaveola A: Perchado junto a un individuo de Tyrannus albogularis. B. Forrajeando en los alrededores del boulevard de Iquitos, Loreto, Perú. Noviembre del 2020. Fotos: Luis A. Garcia. 

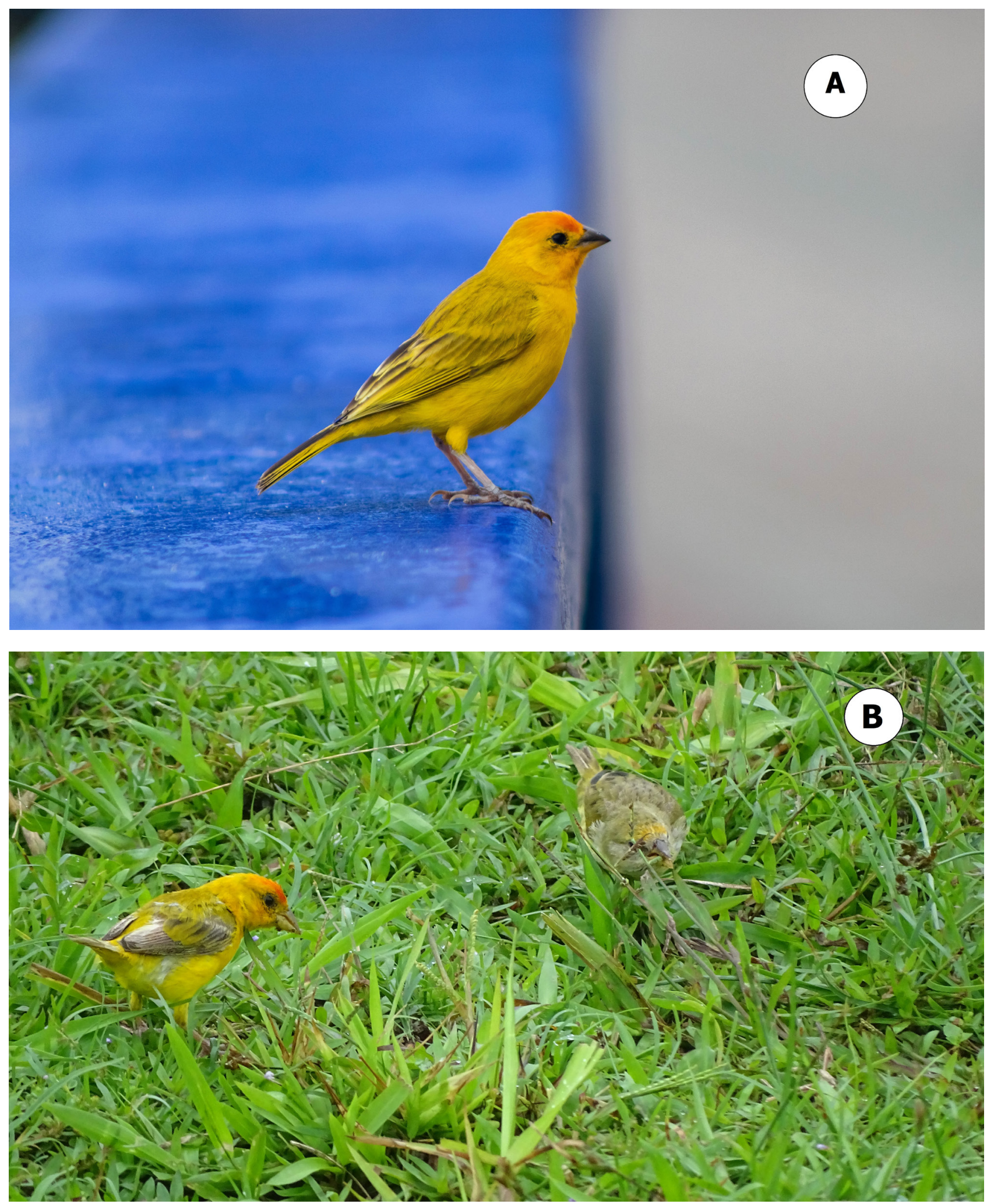

Figura 5. Fotografía de Sicalis flaveola A: Tomando descanso en una baranda del boulevard de Iquitos, Loreto, Perú. Enero del 2021. Foto: Luis A. García. B: Forrajeando en los alrededores de la comunidad nativa de Tres Esquinas, río Putumayo, Loreto, Perú. Abril del 2021. Foto: Freddy R. Ferreyra. 
El 11 de noviembre del 2020, G. A. Tuesta y L. A. Garcia, observaron y fotografiaron a un individuo de $S$. flaveola, a las 08 horas y $24 \mathrm{mi}-$ nutos, en los alrededores del boulevard (Figura $4 \mathrm{~B})$, alimentándose de las semillas de las hierbas que crecen en la parte baja del mencionado lugar.

El 10 de enero del 2021, L. A. Garcia y G. A. Tuesta, observaron y fotografiaron a un individuo de $S$. flaveola, a las 07 horas, en los alrededores del boulevard de Iquitos (Figura $5 \mathrm{C}$ ), en una de las barandas construidas en la zona, aparentemente buscando alimento. Este único individuo observado se desplazaba por toda la zona construida en la parte alta del boulevard, para posteriormente desplazarse hacia la zona baja y desaparecer.

El 22 de abril del 2021, F. R. Ferreyra, observó y fotografió a 7 individuos de $S$. flaveola a las 07 horas, en la comunidad nativa de Tres Esquinas, en el distrito Yaguas, provincia del Putumayo, departamento de Loreto. Este grupo se encontraba alimentándose probablemente de las semillas de las hierbas de la zona, posteriormente se desplazaron con rumbo desconocido (Tabla $1)$.

El 23 de abril del 2021 Freddy Ferreyra, observó y fotografío a 9 individuos de $S$. flaveola a las 08 horas, el grupo también incluía la presencia de dos individuos juveniles (Figura 5 D). Este grupo también fue observado alimentándose de las semillas de las hierbas del lugar, en la comunidad de Tres Esquinas, río Putumayo.

\section{DISCUSIÓN}

Nuestros registros confirman la presencia de Sicalis flaveola en el departamento de Loreto, Perú. S. flaveola estaría ampliando su rango de distribución aproximadamente $341 \mathrm{~km}$ desde la frontera con Colombia en la cuenca baja del río Putumayo (comunidad nativa Tres Esquinas) hacia la ciudad de Iquitos; adicionalmente podemos sugerir que la especie podría estar estableciéndose (residente) en Loreto, por los individuos juveniles observados en esta comunidad. Probablemente los individuos observados en Tres Esquinas, estarían Ilegando desde la zona sur de Colombia, ya que se tiene observaciones de la especie en áreas abiertas del interfluvio de la cuenca media del río Caquetá-Putumayo al sur de la Amazonía Colombiana (Acevedo-Charry et al. 2021), tal como lo están llevando a cabo otras especies recientemente reportadas para Loreto que estarían aprovechando el incremento de espacios abiertos (Piana et al., 2012; Ruiz-Ramos et al., 2017; Ruiz-Ramos et al. 2017; Cuelo, 2018; Ruiz-Ramos et al. 2018; Socolar et al. 2018; Garcia et al., 2020 y Ruiz-Ramos et al. 2020). También se cuenta con registros de la especie introducida en la ciudad fronteriza de Leticia (Colombia) y áreas cercanas a esta ciudad que data del 2006, reportado por Sergio Ocampo-Tobón en la plataforma eBird (https://ebird.org/checklist/ S50317007), probablemente desde esta zona la especie se encuentre desplazándose aguas arriba del río Amazonas.

El registro de un solo individuo de $S$. flaveola en muchas oportunidades en los alrededores de la zona del boulevard en la ciudad de Iquitos, nos podría estar indicando que se trataría de un individuo divagante; aunque no descartamos la posibilidad de que se podría haber escapado de alguna jaula en la ciudad (ya que existe mucho tránsito fluvial entre la ciudad de Leticia y la ciudad de Iquitos), tal como es reportado en algunas ciudades de Sudamérica, incluyendo Lima y Puerto Maldonado (Schulenberg et al., 2010; Guilherme, 2011; Guevara, 2012; Nolazco, 2012; Begazo, 2021). Durante nuestras observaciones desde febrero del 2020 a enero del 2021 solo registramos a un individuo en esta parte de la ciudad, inclusive después del mes de enero se tienen observaciones del individuo hasta el mes marzo del 2021 en la misma zona del boulevard.

Nuestros registros fotográficos descartan que podría tratarse de Sicalis columbiana, ampliamente distribuida en los llanos de Colombia y que también viene siendo observada en algunos sectores del río Amazonas, especialmente 
cercanos a la ciudad de Manaus (Brasil), de acuerdo con algunos registros, por ejemplo el de David Bird en el año 2005, (https://ebird. org/canada/checklist/S32859585); y cuyos detalles en la coloración de la coronilla anterior naranja rufescente la diferencia de su similar $S$. flaveola.

Respecto al comportamiento de $S$. flaveola, en el caso del individuo de Iquitos, se pudo registrar relaciones interespecíficas, especialmente agresiones con otras especies de aves, como Thraupis episcopus, Tyrannus albogularis y Troglodytes aedon. En algunas oportunidades observamos al individuo de $S$. flaveola recolectando y transportando hierbas secas, posiblemente para la construcción de su nido, ya que exploraba lugares adecuados para tal fin, por ejemplo, se observó llevar hierba seca e introducirlo en un tubo de polietileno en la parte alta de un edificio en construcción durante una semana; estuvimos a la espera de registrar a un segundo individuo, pero solo observamos un individuo dedicado a esta labor, este comportamiento fue registrado por cuatro días consecutivos. Y, por último, pudimos notar que el ave es tolerante a la presencia de las personas, ya que no huía cuando se lo fotografiaba a corta distancia.

Podemos concluir que $S$. flaveola estaría presente y quizás residente, ampliando su distribución en la Amazonia de Loreto por la disponibilidad de alimento que encuentra y hábitats favorables para esta especie.

\section{AGRADECIMIENTOS}

A Manuel A. Plenge por la información bibliográfica y orientaciones de las mismas. A Jacob. B. Socolar y Antonio García-Bravo por las valiosas recomendaciones para mejorar la nota científica. A María Isabel Torres Vásquez por la elaboración del mapa distribución en Loreto. Agradecemos a la IUCN por permitir el uso del mapa de distribución de $S$. flaveola.

\section{REFERENCIAS BIBLIOGRÁFICAS}

Acevedo-Chavarry, O., Peña-Alzate, F., Beckers, J., Cabezas, M., Coral-Jaramillo, B., Janni, O., et al. (2021) Avifauna del interfluvio de la cuenca media Caquetá-Putumayo (Japurá-Iça), al sur de la Amazonia colombiana y su respuesta a la huella humana. Revista de la Academia Colombiana de Ciencias Exactas, Fisicas y Naturales. 1-21.

BirdLife International. (2021) Species factsheet: Sicalis flaveola [en línea], Disponible en <http://datazone.birdlife.org/species/ factsheet/saffron-finch-sicalis-flaveola>. [Consulta: 02/07/2021].

Begazo, A (2021) Perú Aves. Saffron Finch Sicalis flaveola. Corbidi, Lima, Perú [en línea], Disponible en <https://www.peruaves. org/thraupidae/saffron-finch-sicalis-flaveola/>. [Consulta: 10/07/2021].

Buitrón, G., Freile, J. (2006) 'Registros inusuales de aves migratorias y de bosques subtropicales en Quito, Ecuador', Cotinga, 26: 54-56.

Cuelo, P. (2018) Primer registro documen $\neg$ tado de Calandria Tropical (Mimus gilvus) para el Perú. Boletín de la Unión de Ornitólogos del Perú, 13(1): 7-9.

eBird. (2021) Una base de datos en línea para la abundancia y distribución de las aves. [en línea], Disponible en <https://ebird. org/home> [Consulta: 2 de julio 2021].

Espinosa, C., Cruz-Bernate, L., Barreto, G. (2017) Biología reproductiva de Sicalis flaveola (AVES: THRAUPIDAE) en Cali, Colombia. Boletín Científico. Centro de Museos. Museo de Historia Natural, 21(2): 101-114.

García, L., Tuesta, C., Ramírez, A., Giardenelli, A., Díaz, A., (2020) Primer registro documentado de la Cigüeña Maguari (Ciconia maguari Gmelin, 1789; Aves: Ciconiidae) en Loreto, Perú, Ciencia Amazónica, 8 (2): 265-272.

Guevara, T. (2012) Avifauna del vivero forestal de la Universidad Nacional Agraria La Molina. Xilema, 25, 83-87. 
Guilherme, E. (2011) Sobre a ocorrencia do canário-da-terra-verdadeiro Sicalis flaveola (Passeriformes, Emberizidae) na Amazonia Sul-ocidental. Atualidades Ornitológicas, 163, 22-23.

IUCN. (2020) The IUCN Red List of Threatened species. (2020) Versión 2020-1. Disponible en: <https://www.iucnredlist.org. Accedi $\neg$ do el 02/07/2021.> [Consulta: 02 julio 2021].

Nolazco, S. (2012) Diversidad de aves silvestres y correlaciones con la cobertura vegetal en parques y jardines de la ciudad de Lima. Boletín de la Unión de Ornitólogos del Perú (UNOP), 7 (1): 4-16.

Silva, G., Pollack, V., Bazán, A. (2012) Avifauna en el campus de la Universidad Nacional de Trujillo-Perú, Mayo - Agosto 2009. UCV-Scientia, 4 (2): 97-203.

Paetzold, V., Querol, E. (2008) Avifauna urbana do município de Uruguaiana, RS, Brasil (resultados parciais). Biodiversidade Pampeana, 6 (1): 40-45.

Piana, R., Díaz, A., Chalco, L., Sevi-llano, C., Saboya, P., Cubas P. (2012) El Caracara Carancho (Caraca $\neg$ ra plancus) estaría expandiendo su rango de distribución hacia el norte y oeste de la Amazonía peruana. Boletín de la Unión de Ornitólogos del Perú (UNOP), 7 (2): 5-11.

Rising, J., Jaramillo, A (2020) Saffron Finch (Sicalis flaveola), versión 1.0. En Birds of the World (J. del Hoyo, A. Elliott, J. Sargatal, DA Christie y E. de Juana, Editores). Laboratorio de Ornitología de Cornell, Ithaca, NY, USA [en línea], Disponible en <https://doi.org/10.2173/bow.saffin.01> [Consulta: 28 mayo 2021].

Ruiz, J. (2002) Registros de Sicalis flaveola: un nuevo residente para Chile. Valdivia, Chile. Boletín Chileno de Ornitología, 9: 30-32.
Ruiz, J. (2007) Nuevos datos sobre la distribución del Chirihue Azafrán (Sicalis flaveola) en la zona centro centro-sur de Chile, Boletín Chileno de Ornitología, 13, 53-55.

Ruiz-Ramos, S., Angulo-Perez, N., Saboya, P., Gaviria, J., Díaz, J. (2020) Registros documentados del Corocoro Escarlata (Eudocimus ruber Linnaeus, 1758; Aves: Threskiornithidae) en Loreto, Perú. Ciencia Amazónica, 8 (1): 123-130.

Ruiz-Ramos, S., Alegría-Torres, B., Ayapi-Da-Silva, J., Díaz-Alván, J. (2017) Registro documentado del Avefría Tero (Vanellus chilensis) en selva norte del Perú. Boletín de la Unión de Ornitólogos del Perú (UNOP), 12 (1): 15-18.

Ruiz-Ramos, S., Ayapi-Da-Silva, J., Alegría-Torres, B., Díaz, J. (2018) Registro documentado del Zambullidor Menor (Tachybaptus dominicus) en la selva norte de Loreto. Boletín de la Unión de Ornitólogos del Perú (UNOP), 13 (2): 12-15.

Schulenberg, T., Stotz, D., Lane, D., O'Neill, J., Parker, T. (2010) Birds of Peru. Revised and updated edition. Princeton, NJ: Princeton University Press.

Socolar, J., Díaz-Alván. J., Saboya, P., Pomara, L., O'Shea, B., Cubas, P. et al. (2018) Noteworthy bird records from northeastern Peru reveal connectivity and isolation in the western Amazonian avifauna. The WiIson Journal of Ornithology, 130, 94-111

\section{Conflicto de interés}

Los autores declaramos no tener ningún conflicto de interés. 